\title{
Swyer-James-McLeod Syndrome
}

\author{
Esther Molnar, MD, Arun Matthew, MD
}

Admission chest x-ray of an 81 year-old man admitted to the hospital for atrial fibrillation with rapid ventricular response is shown in Figure 1. The chest $x$-ray shows a unilateral hyperlucent lung, with paucity of peripheral vessels and decreased lung volume. Differential diagnosis for unilateral hyperlucent lung includes Swyer-James-McLeod syndrome (SJMS), centrilobular emphysema, allergic bronchopulmonary aspergillosis, congenital lobar over inflation, bronchial atresia and congenital interruption of pulmonary artery. ${ }^{1}$ High resolution CT imaging confirmed the diagnosis of SJMS in this patient.

SJMS is a manifestation of post-infectious obliterative bronchiolitis. SJMS was first described in 1953 as a unilateral hyperlucent, hypovascular lung with a small ipsilateral hilum. Bronchiectasis, bronchial wall thickening and small pulmonary arteries are commonly associated findings. SJMS is thought to occur from severe post-infectious bronchiolitis occurring at less than eight years of age. The damage to the airways in early childhood impairs normal development of the alveolar ducts and leads to diminished arterial flow and hypoplastic pulmonary vasculature. Terminal air sacs distal to areas of bronchiolar obstruction become hyperinflated and alveoli are overdistended, adding to resistance of blood flow and giving the appearance of radiographic hyperlucency. The most common etiology of this phenomenon is adenovirus infection, but other infectious causes include paramyxovirus, Bordetella pertussis, mycobacterium tuberculosis, mycoplasma pneumoniae, influenza A or respiratory syncytial virus infections. The prevalence of SJMS has been reported as less than $0.01 \%$ in the general population. ${ }^{2,3}$

To make the diagnosis of SJMS, available modalities include ventilation-perfusion scintigraphy (V/Q scan) and $C T$ imaging. V/Q scanning supports the diagnosis with matched ventilation/perfusion defects. ${ }^{4}$ High resolution CT (HRCT) during forced expiration is useful in demonstrating air trapping. The appearance of the lung on HRCT expiration with mosaic pattern attenuation and lack of change in volume with expiration is essential in making the diagnosis. HRCT is also useful in ruling out other potential causes of unilateral hyperlucent lung, such as bronchial atresia. 5.6

SJMS is most commonly diagnosed by the fourth decade of life, usually as an incidental finding. Symptoms tend to be widely varied, ranging from completely asymptomatic patients to those with wheezing, coughing, hemoptysis, dyspnea on exertion, and recurrent bronchopulmonary infections. Management of SJMS includes early treatment of infections and influenza and pneumococcal vaccination. Bronchodilators may be helpful to patients demonstrating obstructive defects on spirometry. For severe cases with recurrent bronchopulmonary infections, lobectomy or pneumonectomy is helpful. ${ }^{2}$

\section{REFERENCES}

1. Garg R, Aga P, Saheer S, Jabeed P, Singh A, Hassan G, et al. Swyer-james-MacLeod syndrome with ipsilateral herniation of hyperinflated hyperlucent lung. BMJ Case Rep. 2011 Aug 31; 2011 : 10.1136/bcr.05.2011.4191.

2. Fregonese L, Girosi D, Battistini E, Fregonese B, Risso FM, Bava GL, et al. Clinical, physiologic, and roentgenographic changes after pneumonectomy in a boy with macleod/swyer-james syndrome and bronchiectasis. Pediatr Pulmonol. 2002 Nov; 34(5): 412-6.

3. Dillman JR, Sanchez R, Ladino-Torres MF, Yarram SG, Strouse PJ, Lucaya J. Expanding upon the unilateral hyperlucent hemithorax in children. Radiographics. 2011 May-Jun; 31(3): 723-41.

4. Chen IC, Chen YW, Lin SH, Hsu JH, Wu JR, Dai ZK. Usefulness of combination of pulmonary ventilation and perfusion scintigraphy on the diagnosis of children with unilateral hyperlucent lung. $\mathrm{Nucl}$ Med Commun. 2011 Nov; 32(11): 1052-9.

5. Arslan N, Ilgan S, Ozkan M, Yuksekol I, Bulakbasi N, Pabuscu Y, et al. Utility of ventilation and perfusion scan in the diagnosis of young military recruits with an incidental finding of hyperlucent lung. Nucl Med Commun. 2001 May; 22(5): 525-30.

6. OGhossain MA, Achkar A, Buy JN, Rochemaure J, Vadrot D. Swyer-james syndrome documented by spiral CT angiography and high resolution inspiratory and expiratory CT: An accurate single modality exploration. J Comput Assist Tomogr. 1997 Jul-Aug; 21(4): 616-8. 


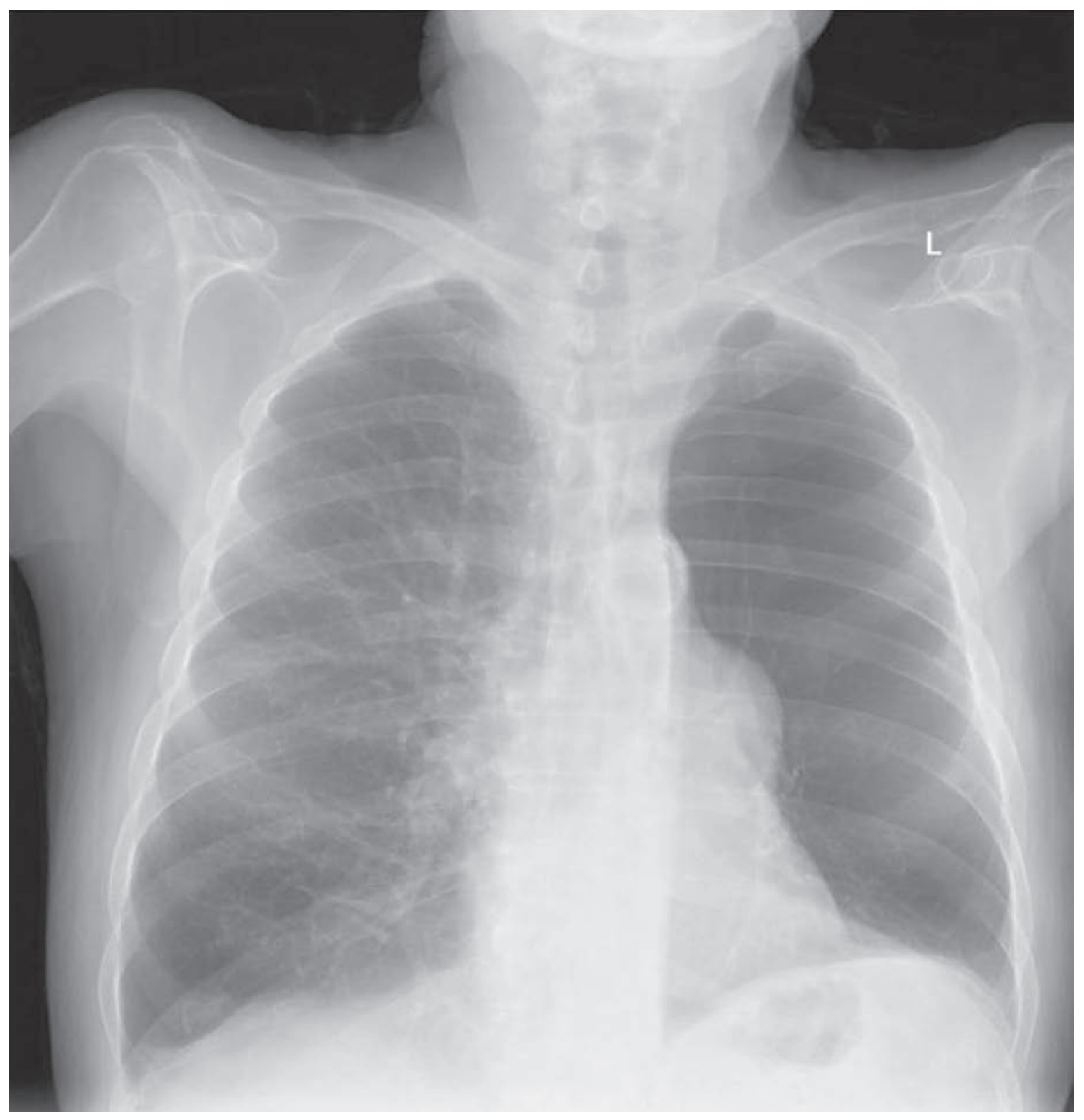

Figure 1: Admission chest X-ray

The Medicine Forum | 71 